PROCEEDINGS OF THE

AMERICAN MATHEMATICAL SOCIETY

Volume 130, Number 5, Pages 1437-1445

S 0002-9939(01)06211-6

Article electronically published on October 12, 2001

\title{
A UNIQUENESS RESULT CONCERNING SCHUR IDEALS
}

\author{
JAMES P. SOLAZZO
}

(Communicated by Joseph A. Ball)

\begin{abstract}
A subset of the set of all positive semi-definite matrices of a given size which is invariant under Schur (componentwise) multiplication by an arbitrary positive semi-definite matrix is said to be a Schur ideal. A subset of $k$-dimensional complex space is said to be hyperconvex if it arises as the set of possible values $\left(w_{1}, \ldots, w_{k}\right)=\left(f\left(\alpha_{1}\right), \ldots, f\left(\alpha_{k}\right)\right)$ arising from restricting contractive elements $f$ from some uniform algebra $A$ to a finite set $\left\{\alpha_{1}, \ldots, \alpha_{k}\right\}$ in the domain. When the uniform algebra is the disk algebra, the hyperconvex set is said to be a Pick body. Motivated by the classical Pick interpolation theorem, Paulsen has introduced a natural notion of duality between Schur ideals and hyperconvex sets. By using some recently developed results in operator algebras (matricial Schur ideals), we show that each Pick body has a unique affiliated Schur ideal.
\end{abstract}

\section{INTRODUCTION}

The classical Nevanlinna-Pick problem is the following: given $\left\{\alpha_{1}, \ldots, \alpha_{k}\right\}$ a $k$ tuple of distinct points with $\left|\alpha_{i}\right|<1$ and given $\left\{w_{1}, \ldots, w_{k}\right\}$ a $k$-tuple of complex numbers, what are the necessary and sufficient conditions for the existence of a function $f$, analytic in the open disk $\mathbb{D},\|f\|_{\infty} \leq 1$ and such that $f\left(\alpha_{i}\right)=w_{i}$ ? When such a function $f$ exists, the function $f$ is said to interpolate the given sequences $\left\{\alpha_{i}\right\}$ and $\left\{w_{i}\right\}$. This question was first answered by G. Pick in 1916 in his paper 11] where he states explicitly when one can interpolate the given sequences $\left\{\alpha_{i}\right\}$ and $\left\{w_{i}\right\}$. In 1919 (1929) R. Nevanlinna contributed to the study of interpolation by bounded analytic functions in his paper(s) [7 (8]) by providing an inductive procedure which enables one to construct a bounded analytic function in $\mathbb{D}$ which interpolates the given sequences $\left\{\alpha_{i}\right\}$ and $\left\{w_{i}\right\}$. We will now state Pick's theorem.

Theorem 1. If $\alpha_{1}, \ldots, \alpha_{k}, w_{1}, \ldots, w_{k} \in \mathbb{D}$, then there exists an analytic function $f: \mathbb{D} \rightarrow \mathbb{D}$ with $f\left(\alpha_{i}\right)=w_{i}$ if and only if the matrix

$$
\left(\frac{1-\bar{w}_{i} w_{j}}{1-\bar{\alpha}_{i} \alpha_{j}}\right)_{i, j=1}^{k}
$$

is positive semi-definite.

Fix $\alpha_{1}, \ldots, \alpha_{k}$ in $\mathbb{D}$. B. Cole, K. Lewis, and J. Wermer in [4] define a Pick body, denoted $\mathcal{P}\left(\alpha_{1}, \ldots, \alpha_{k}\right)$, as follows:

$$
\mathcal{P}\left(\alpha_{1}, \ldots, \alpha_{k}\right)=\left\{\vec{w} \in \mathbb{C}^{k}: \exists f \in H^{\infty}(\mathbb{D}),\|f\|_{\infty} \leq 1, f\left(\alpha_{i}\right)=w_{i}\right\} .
$$

Received by the editors October 4, 2000 and, in revised form, November 20, 2000.

2000 Mathematics Subject Classification. Primary 47A57, 46L07; Secondary 47L25, 47L30. 
This is precisely the set of all $k$-tuples $\left(w_{1}, \ldots, w_{k}\right)$ in $\mathbb{C}^{k}$ which satisfy the necessary and sufficient conditions of Pick's theorem. It turns out that Pick bodies are a special class of sets contained in a more general class of sets called "hyperconvex". To this end, let $X$ be a compact Hausdorff space and let $C(X)$ denote the continuous complex-valued functions on $X$. We will call $A \subseteq C(X)$ a uniform algebra provided that $A$ is closed, contains the identity, and separates points in $X$. Fix $x_{1}, \ldots, x_{k}$ in $X$. B. Cole, K. Lewis, and J. Wermer in [4] define an interpolation body, denoted $\mathcal{D}\left(A ; x_{1}, \ldots, x_{k}\right)$, in the following way: a point $\vec{w}=\left(w_{1}, \ldots, w_{k}\right)$ in $\mathbb{C}^{k}$ belongs to $\mathcal{D}\left(A ; x_{1}, \ldots, x_{k}\right)$ if for each $\epsilon>0$, there exists $f$ in $A$ with $\|f\|_{\infty} \leq 1+\epsilon$ such that $f\left(x_{i}\right)=w_{i}$. The set $\mathcal{D}\left(A ; x_{1}, \ldots, x_{k}\right)$ is the unit ball of the Banach algebra $\mathbb{C}^{k}$ (under coordinate-wise multiplication) for some norm on $\mathbb{C}^{k}$. Sets in the closed $k$-polydisk arising as $\mathcal{D}\left(A ; x_{1}, \ldots, x_{k}\right)$ for some $A$ and $x_{1}, \ldots, x_{k}$ in $X$ are called hyperconvex in 4 . If we let $A(\mathbb{D})$ denote the analytic functions in $\mathbb{D}$ which are continuous on $\mathbb{D}^{-}$and let $\alpha_{1}, \ldots, \alpha_{k}$ in $\mathbb{D}$, then in 4 they show that the Pick body $\mathcal{P}\left(\alpha_{1}, \ldots, \alpha_{k}\right)=\mathcal{D}\left(A(\mathbb{D}) ; \alpha_{1}, \ldots, \alpha_{k}\right)$.

Schur ideals are a relatively new concept which act as a natural "dual" object for hyperconvex sets. A precise definition of the Schur ideal will be given in Section 2. In general, there are often many Schur ideals that could serve as the "dual" of a hyperconvex set. In this paper we shall show that for this special class of hyperconvex sets (i.e., Pick bodies) there is a unique "dual" Schur ideal.

\section{Preliminaries}

Let $A$ contained in $C(X)$ be a uniform algebra and let $I_{x}$ denote the ideal of functions vanishing at the points $x_{1}, \ldots, x_{k}$ in $X$. Then the quotient algebra $\mathcal{A}=A / I_{x}$ is an operator algebra [3. Since $A$ separates points in $X$ there exist functions $f_{1}, \ldots, f_{k}$ in $A$ with $f_{i}\left(x_{j}\right)=\delta_{i, j}$ where $\delta_{i, j}$ denotes the Kronecker delta. Thus in $\mathcal{A}$ we have that $E_{i}=\left[f_{i}\right]$ are a family of $k$ commuting idempotents satisfying $E_{i} E_{j}=\delta_{i, j} E_{j}, E_{1}+\ldots+E_{k}=1$, and which span $\mathcal{A}$. Therefore, when studying the properties of an interpolation body, one can choose to work with the uniform algebra $A$ or its corresponding quotient algebra (operator algebra) $\mathcal{A}$ since we have the following equalities:

$$
\begin{aligned}
\mathcal{D}\left(A ; x_{1}, \ldots, x_{k}\right)= & \left\{\vec{w} \in \mathbb{C}^{k}: \exists f \in A,\|f\| \leq 1 \text { and } f\left(x_{i}\right)=w_{i}\right\}^{-} \\
= & \left\{\vec{w} \in \mathbb{C}^{k}: \exists f \in A,\|[f]\| \leq 1 \text { and } f\left(x_{i}\right)=w_{i}\right\} \\
= & \left\{\left(w_{1}, \ldots, w_{k}\right) \in \mathbb{C}^{k}:\left\|w_{1} E_{1}+\ldots+w_{k} E_{k}\right\| \leq 1\right\} .
\end{aligned}
$$

For this reason we will abbreviate $\mathcal{D}\left(A ; x_{1}, \ldots, x_{k}\right)$ as $\mathcal{D}(\mathcal{A})$ and often interchange the two without mention.

Recall if we let $M_{n}$ denote the $C^{*}$-algebra of $n \times n$ matrices and let $M_{n}(A)$ denote the algebra of $n \times n$ matrices over $A$, then $M_{n}(A)$ is endowed with the norm

$$
\|F\|=\sup \left\{\|F(x)\|_{M_{m}}: x \in X\right\},
$$

where $F=\left(f_{i j}\right)$ is in $M_{n}(A)$. Moreover if $x_{1}, \ldots, x_{k}$ in $X$ and $\mathcal{A}=A / I_{x}$, then $M_{n}(\mathcal{A})$ is endowed with the quotient norm by identifying

$$
M_{n}(\mathcal{A})=M_{n}(A) / M_{n}\left(I_{x}\right) .
$$


Now given points $x_{1}, \ldots, x_{k}$ in $X$, the abstract interpolation problem is concerned with determining the set of $k$-tuples $\vec{W}=\left(W_{1}, \ldots, W_{k}\right)$ of $n \times n$ matrices, denoted

$$
\begin{aligned}
\mathcal{D}_{n}\left(A ; x_{1}, \ldots, x_{k}\right) & =\left\{\vec{W}: \exists F \in M_{n}(A),\|F\| \leq 1, F\left(x_{i}\right)=w_{i}\right\}^{-} \\
& =\left\{\vec{W}: \exists F \in M_{n}(A),\|[F]\| \leq 1, F\left(x_{i}\right)=w_{i}\right\} \\
& =\left\{\left(W_{1}, \ldots, W_{k}\right):\left\|\sum_{i=1}^{k} W_{i} \otimes E_{i}\right\| \leq 1\right\} .
\end{aligned}
$$

Thus the sets $\left\{\mathcal{D}_{n}(\mathcal{A})\right\}, n=1,2, \ldots$, are just a natural coordinatization of the closed unit balls of $M_{n}(\mathcal{A})$ for an operator algebra $\mathcal{A}$ generated by $k$-idempotents satisfying the above relations.

Throughout the paper $M_{k}$ will denote the $k \times k$ matrices with complex entries and $M_{k}^{+}$will denote the closed cone of positive semi-definite matrices in $M_{k}$. If $P$ is in $M_{k}$, then it will be understood that $P$ is the matrix $\left(p_{i j}\right)_{i, j=1}^{k}$. If $P, Q$ are in $M_{k}$, then the Schur product of the matrices $P$ and $Q$, denoted as $P * Q$, is defined to be $\left(p_{i j}\right) *\left(q_{i j}\right)=\left(p_{i j} q_{i j}\right)$.

The following theorem is due to Cole and Wermer [5], in which they give conditions on a point $\left(w_{1}, \ldots, w_{k}\right)$ in $\mathbb{C}^{k}$ in order that $\left(w_{1}, \ldots, w_{k}\right)$ belong to the interpolation body $\mathcal{D}\left(A ; x_{1}, \ldots, x_{k}\right)$ where $A$ is an arbitrary uniform algebra.

Theorem 2. If $A \subseteq C(X)$ is a uniform algebra and $x_{1}, \ldots, x_{k} \in X$, then there exists a set $\mathcal{S} \subseteq M_{k}^{+}$such that

$$
\left(w_{1}, \ldots, w_{k}\right) \in \mathcal{D} \text { if and only if }\left(\left(1-\bar{w}_{i} w_{j}\right) p_{i j}\right) \geq 0 \text { for all }\left(p_{i j}\right) \in \mathcal{S} \text {. }
$$

In [9], V. Paulsen observes that Theorem 2 suggests the following "dualities" between closed subsets of the closed $k$-polydisk and subsets of $M_{k}^{+}$. Given a nonempty set $\mathcal{S} \subseteq M_{k}^{+}$define

$$
\mathcal{S}^{\perp}=\left\{\left(w_{1}, \ldots, w_{k}\right) \in \mathbb{C}^{k}:\left(\left(1-\bar{w}_{i} w_{j}\right) p_{i j}\right) \geq 0 \forall\left(p_{i j}\right) \in \mathcal{S}\right\} .
$$

Similarly, given a subset $\mathcal{D}$ of the closed $k$-polydisk with $0 \in \mathcal{D}$ define

$$
\mathcal{D}^{\perp}=\left\{\left(p_{i j}\right):\left(\left(1-\bar{w}_{i} w_{j}\right) p_{i j}\right) \geq 0 \forall\left(w_{1}, \ldots, w_{k}\right) \in \mathcal{D}\right\} .
$$

Since we insist that $0 \in \mathcal{D}$, then $\mathcal{D}^{\perp}$ is always a set of positive semi-definite matrices. Observing further that the set $\mathcal{D}^{\perp}$ has certain properties, V. Paulsen [9] introduces the concept of a Schur ideal. By studying this duality between hyperconvex sets and Schur ideals and using some results from the theory of abstract operator algebras, V. Paulsen is able to generalize J. Agler's scalar-valued interpolation results [1] for the bidisk to more general product domains [9]. For another approach to such problems see the paper of A. Tomerlin [14].

Definition 1. Let $\mathcal{I} \subseteq M_{k}{ }^{+}$be a non-empty set. Then $\mathcal{I}$ will be called a Schur ideal provided that:

(1) $A, B \in \mathcal{I} \Rightarrow A+B \in \mathcal{I}$.

(2) $A \in \mathcal{I}, P \in M_{k}{ }^{+} \Rightarrow A * P \in \mathcal{I}$.

If $\mathcal{D}$ is a subset of the closed $k$-polydisk with $0 \in \mathcal{D}$, then $\mathcal{D}^{\perp}$ is a Schur ideal. Moreover if $\mathcal{D}$ is a hyperconvex set, then $\mathcal{D}=\mathcal{D}^{\perp \perp}$.

Definition 2. Let $A \subseteq C(X), x_{1}, \ldots, x_{k} \in X$, and $\mathcal{S} \subseteq M_{k}^{+}$. Then the set $\mathcal{S}$ is said to be affiliated with the interpolation body $\mathcal{D}\left(A ; x_{1}, \ldots, x_{k}\right)$ provided that $\mathcal{S}^{\perp}=\mathcal{D}\left(A ; x_{1}, \ldots, x_{k}\right)$. 
Note that each interpolation body $\mathcal{D}\left(A ; x_{1}, \ldots, x_{k}\right)$ has an affiliated set (Schur ideal), namely $\mathcal{D}\left(A ; x_{1}, \ldots, x_{k}\right)^{\perp}$.

Example. Fix $\alpha_{1}, \ldots, \alpha_{k}$ in $\mathbb{D}$ and let $P=\left(\frac{1}{1-\bar{\alpha}_{i} \alpha_{j}}\right)$. Note that in Theorem 2 if $A=A(\mathbb{D})$ and $x_{i}=\alpha_{i}$, then $\mathcal{S}=\{P\}$. It follows from Theorem 1 that $\mathcal{P}\left(\alpha_{1}, \ldots, \alpha_{k}\right)=\{P\}^{\perp}$. However if $v_{1}, \ldots, v_{k}$ in $\mathbb{C}^{k}$ such that $v_{i} \neq 0$ and $\left(w_{1}, \ldots, w_{k}\right)$ in $\{P\}^{\perp}$, then we have that

$$
\begin{aligned}
\left(\frac{1-\bar{w}_{i} w_{j}}{1-\bar{\alpha}_{i} \alpha_{j}}\right) \geq 0 & \Longrightarrow D_{v}^{*}\left(\frac{1-\bar{w}_{i} w_{j}}{1-\bar{\alpha}_{i} \alpha_{j}}\right) D_{v} \geq 0 \\
& \Longrightarrow\left(1-\bar{w}_{i} w_{j}\right) * D_{v}^{*}\left(\frac{1}{1-\bar{\alpha}_{i} \alpha_{j}}\right) D_{v} \geq 0 \\
& \Longrightarrow\left(w_{1}, \ldots, w_{k}\right) \in\left\{D_{v}^{*} P D_{v}\right\}^{\perp} .
\end{aligned}
$$

Similarly if $\left(w_{1}, \ldots, w_{k}\right)$ in $\left\{D_{v}^{*} P D_{v}\right\}^{\perp}$, then $\left(w_{1}, \ldots, w_{k}\right)$ in $\{P\}^{\perp}$. Here $D_{v}$ denotes the matrix with diagonal entries $v_{1}, \ldots, v_{k}$ and zeros elsewhere.

Thus, given an interpolation body $\mathcal{D}$, an affiliated set $\mathcal{S}$ need not be unique. This is why we study Schur ideals. In this example we are simply producing different generating sets for the same Schur ideal. This is why the "right" question is whether or not there is a unique Schur ideal affiliated with a given hyperconvex set. For Pick bodies we have uniqueness. We now state our main result.

Theorem A. If $\mathcal{I} \subseteq M_{k}^{+}$is a closed Schur ideal such that $\mathcal{I}^{\perp}=\mathcal{P}\left(\alpha_{1}, \ldots, \alpha_{k}\right)$, then $\mathcal{I}=\left\langle\left(\frac{1}{1-\overline{\alpha_{i}} \alpha_{j}}\right)\right\rangle$.

Here $\left\langle\left(\frac{1}{1-\overline{\alpha_{i}} \alpha_{j}}\right)\right\rangle$ denotes the Schur ideal generated by the matrix $\left(\frac{1}{1-\overline{\alpha_{i}} \alpha_{j}}\right)$ (i.e, $\left.\left(\frac{1}{1-\overline{\alpha_{i} \alpha_{j}}}\right) * M_{k}^{+}\right)$. So despite the fact that the Pick body $\mathcal{P}\left(\alpha_{1}, \ldots, \alpha_{k}\right)$ does not have a unique affiliated set $\mathcal{S} \subseteq M_{k}^{+}$, it does have a unique affiliated Schur ideal. Theorem A can really be thought of as just a matrix theory result. However, it is surprising that for a proof we need to invoke the abstract theory of operator algebras (matricial Schur ideals) to solve this matrix theory problem. The reader is encouraged to try to find an alternate proof. By solving this problem we are able to gain some insight into understanding the following problem:

Problem. Let $\mathcal{I} \subseteq M_{k}^{+}$be a closed Schur ideal such that

$$
\begin{aligned}
\mathcal{I}^{\perp}=\mathcal{D}\left(A\left(\mathbb{D}^{2}\right) ;\left(\alpha_{1}, \beta_{1}\right), \ldots,\left(\alpha_{k}, \beta_{k}\right)\right) . \\
I s \mathcal{I}=\left\langle\left(\frac{1}{1-\overline{\alpha_{i}} \alpha_{j}}\right)\right\rangle \cap\left\langle\left(\frac{1}{1-\beta_{i} \beta_{j}}\right)\right\rangle ?
\end{aligned}
$$

In joint work with V. Paulsen and G. Weiss, we have shown that for three particular points, say $\left(\alpha_{1}, \beta_{1}\right),\left(\alpha_{2}, \beta_{2}\right),\left(\alpha_{3}, \beta_{3}\right)$ in the bidisk, $\left\langle\left(\frac{1}{1-\overline{\alpha_{i}} \alpha_{j}}\right)\right\rangle \cap\left\langle\left(\frac{1}{1-\beta_{i} \beta_{j}}\right)\right\rangle$ is not finitely generated. Note that

$$
\mathcal{D}\left(A\left(\mathbb{D}^{2}\right) ; z_{1}, \ldots, z_{k}\right)^{\perp}=\left\langle\left(\frac{1}{1-\bar{\alpha}_{i} \alpha_{j}}\right)\right\rangle \cap\left\langle\left(\frac{1}{1-\bar{\beta}_{i} \beta_{j}}\right)\right\rangle ;
$$

see [9]. If the above Problem is answered in the affirmative, then this would show that J. Agler's bidisk formula [1] is not computable in the following sense. 
Definition 3. The set $\mathcal{D}\left(A ; x_{1}, \ldots, x_{k}\right)$ is said to be weakly computable provided that there exists a finitely generated Schur ideal $\mathcal{I}$ with $\mathcal{I}^{\perp}=\mathcal{D}\left(A ; x_{1}, \ldots, x_{k}\right)$.

\section{Matricial Schur ideals}

In this section we give a survey of those results in V. Paulsen's paper, Operator Algebras of Idempotents [10] (preprint), necessary to prove Theorem A. Since this is just a survey, the proofs will be omitted. The theorem (lemma, proposition, and definition) numbers in parentheses coincide with the numbers in [10].

Definition 4. (1) Fix a natural number $k$. A sequence of sets $\mathcal{S}=\left\{\mathcal{S}_{n}\right\}, \mathcal{S}_{n} \subseteq$ $M_{k}\left(M_{n}\right)^{+}$will be called a matricial Schur ideal provided that:

(i) $\left(Q_{i j}\right),\left(P_{i j}\right) \in \mathcal{S}_{n} \Rightarrow\left(Q_{i j}+P_{i j}\right) \in \mathcal{S}_{n}$.

(ii) $\left(Q_{i j}\right) \in \mathcal{S}_{n}$ and $B_{1}, \ldots, B_{k}$ are $n \times m$ matrices $\Rightarrow\left(B_{i}^{*} Q_{i j} B_{j}\right) \in \mathcal{S}_{m}$.

Let $E_{1}, \ldots, E_{k}$ be bounded operators on a Hilbert space $\mathcal{H}$ satisfying $E_{i} E_{j}=$ $\delta_{i, j} E_{i}$ and $E_{1}+\ldots+E_{k}=I$. We let $\mathcal{A}$ denote the (matrix-normed) operator algebra generated by this set. We shall call such an algebra a $k$-idempotent algebra. Given a concrete $k$-idempotent algebra on a Hilbert space, let $\mathcal{A}^{*} \mathcal{A}=\operatorname{span}\left\{E_{i}^{*} E_{j}\right.$ : $i, j=1, \ldots, k\}$ denote the corresponding operator system.

Lemma 1. (2.1) Let $A_{i j} \in M_{n}$. Then $\sum_{i, j=1}^{k} A_{i j} \otimes E_{i}^{*} E_{j} \geq 0$ on $\mathbb{C}^{n} \otimes \mathcal{H}$ if and only if $\left(A_{i j} \otimes E_{i}^{*} E_{j}\right) \geq 0$ on $\mathbb{C}^{n k} \otimes \mathcal{H}$.

Definition 5. (2) Let $\mathcal{A}$ be a $k$-idempotent algebra. Set

$$
\mathcal{S}_{n}\left(\mathcal{A}^{*} \mathcal{A}\right)=\left\{\left(\Phi\left(E_{i}^{*} E_{j}\right)\right): \Phi: \mathcal{A}^{*} \mathcal{A} \rightarrow M_{n} \text { is completely positive }\right\} .
$$

Theorem 3. (2.2) Let $\mathcal{A}$ be a k-idempotent algebra. Then:

(1) $\mathcal{S}\left(\mathcal{A}^{*} \mathcal{A}\right)=\left\{\mathcal{S}_{n}\left(\mathcal{A}^{*} \mathcal{A}\right)\right\}$ is a matricial Schur ideal.

(2) Let $A_{i j} \in M_{n}, i, j=1, \ldots, k$. Then $\left(A_{i j} \otimes E_{i}^{*} E_{j}\right) \geq 0$ if and only if $\left(A_{i j} \otimes Q_{i j}\right) \geq$ 0 for all $\left(Q_{i j}\right) \in \mathcal{S}_{n}\left(\mathcal{A}^{*} \mathcal{A}\right)$.

(3) Let $\mathcal{B}=\operatorname{span}\left\{F_{i}: i=1, \ldots, k\right\}$ be another $k$-idempotent algebra. Then the map $E_{i}^{*} E_{j} \rightarrow F_{i}^{*} F_{j}$ is n-positive if and only if $\mathcal{S}_{n}\left(\mathcal{B}^{*} \mathcal{B}\right) \subseteq \mathcal{S}_{n}\left(\mathcal{A}^{*} \mathcal{A}\right)$.

Definition 6. (3) Let $\mathcal{A}$ be a $k$-idempotent algebra. Set $\mathcal{S}_{n}(\mathcal{A})=\left\{\left(Q_{i j}\right) \in\right.$ $M_{k}\left(M_{n}\right)^{+}:\left(\left(I-W_{i}^{*} W_{j}\right) \otimes Q_{i j}\right) \geq 0 \forall\left(W_{1}, \ldots, W_{k}\right) \in \mathcal{D}_{m}(\mathcal{A}), m$ arbitrary $\}$.

Theorem 4. (2.3) Let $\mathcal{A}$ be a k-idempotent algebra. Then:

(1) $\mathcal{S}(\mathcal{A})=\left\{\mathcal{S}_{n}(\mathcal{A})\right\}$ is a matricial Schur ideal.

(2) Let $W_{i} \in M_{n}$. Then $\left\|\sum W_{i} \otimes E_{i}\right\| \leq 1$ if and only if $\left(\left(I_{n}-W_{i}^{*} W_{j}\right) \otimes Q_{i j}\right) \geq 0$ for all $\left(Q_{i j}\right) \in \mathcal{S}_{n}(\mathcal{A})$.

(3) $\mathcal{S}_{n}\left(\mathcal{A}^{*} \mathcal{A}\right) \subseteq \mathcal{S}_{n}(\mathcal{A})$ for all $n$. Let $\mathcal{B}=\operatorname{span}\left\{F_{i}: i=1, \ldots, k\right\}$ be another $k$-idempotent algebra.

(4) The following are equivalent:

(i) $E_{i} \rightarrow F_{i}$ is $n$ contractive.

(ii) $\mathcal{D}_{n}(\mathcal{A}) \subseteq \mathcal{D}_{n}(\mathcal{B})$.

(iii) $\mathcal{S}_{n}(\mathcal{B}) \subseteq \mathcal{S}_{n}(\mathcal{A})$.

Consequently, the map $E_{i} \rightarrow F_{i}$ is a completely isometric isomorphism if and only if $\mathcal{S}(\mathcal{A})=\mathcal{S}(\mathcal{B})$. 
Let $P$ in $M_{k}^{+}$with $p_{i j} \neq 0$ for all $i$ and $j$ and define $\mathcal{A}(P)=\left\{E_{i}=P^{1 / 2} E_{i i} P^{-1 / 2}\right.$ : $i=1, \ldots, k\}$ where $E_{i j}$ denote the canonical matrix units. In Example 2.4 [10], V. Paulsen shows that

$$
\mathcal{S}_{n}\left(\mathcal{A}(P)^{*} \mathcal{A}(P)\right)=\left\{\left(p_{i j} R_{i j}\right):\left(R_{i j}\right) \in M_{k}\left(M_{n}\right)^{+}\right\}
$$

In particular, $\mathcal{S}_{1}\left(\mathcal{A}(P)^{*} \mathcal{A}(P)\right)=\langle P\rangle$. In Example 2.6 [10, it is shown when $P$ is the special matrix $\left(\frac{1}{1-\bar{\alpha}_{i} \alpha_{j}}\right)$ that $\mathcal{S}_{n}(\mathcal{A}(P))=\mathcal{S}_{n}\left(\mathcal{A}(P)^{*} \mathcal{A}(P)\right)$ for all $n$. Moreover when $P=\left(\frac{1}{1-\bar{\alpha}_{i} \alpha_{j}}\right)$, every isometric representation of $\mathcal{A}(P)$ is completely isometric. One can see this by utilizing the matrix-valued Nevanlinna-Pick Theorem 12 from which one can verify that $\mathcal{A}(P)$ is completely isometrically isomorphic to $A(\mathbb{D}) / I_{\alpha}$. Thus if $\mathcal{B}$ is isometrically isomorphic to $\mathcal{A}(P)$, then $\mathcal{B}$ is isometrically isomorphic to $A(\mathbb{D}) / I_{\alpha}$. Hence by a theorem of $\mathrm{W}$. Arveson 2 , $\mathcal{B}$ is completely isometrically isomorphic to $A(\mathbb{D}) / I_{\alpha}$ and the result follows. We close this section with the following proposition.

Proposition 5. (2.8) Let $\mathcal{S} \subseteq M_{k}\left(M_{n}\right)^{+}$satisfy $\left(Q_{i j}\right) \in \mathcal{S}$ implies $\left(B_{i}^{*} Q_{i j} B_{j}\right) \in \mathcal{S}$ whenever $B_{1}, \ldots, B_{k}$ are in $M_{n}$, and let $A_{i j} \in M_{n}$. Then $\left(A_{i j} \otimes Q_{i j}\right) \geq 0$ for all $\left(Q_{i j}\right) \in \mathcal{S}$ if and only if $\sum_{i, j=1}^{k} \operatorname{Tr}\left(A_{i j}^{t} Q_{i j}\right) \geq 0$ for all $\left(Q_{i j}\right) \in \mathcal{S}$, where $\operatorname{Tr}$ denotes the trace of the matrix and $A^{t}$ denotes the transpose of the matrix $A$.

\section{Proof of Theorem A}

Before proving Theorem A we will state and prove three lemmas from which the proof of Theorem A will follow. The following notation will remain fixed for the remainder of the paper.

Fix $\alpha_{1}, \ldots, \alpha_{k}$ in $\mathbb{D}$. Let $\mathcal{I} \subseteq M_{k}^{+}$such that $\mathcal{I}^{\perp}=\mathcal{P}\left(\alpha_{1}, \ldots, \alpha_{k}\right)$. Let $P=$ $\left(\frac{1}{1-\overline{\alpha_{i}} \alpha_{j}}\right)$ and define $\mathcal{A}(P)$ as in Section 3. If we let $\mathcal{R}=\{Q \in \mathcal{I}: Q$ is invertible $\}$, then $\langle\mathcal{R}\rangle^{-}=\mathcal{I}$ since $\mathcal{I}^{\perp}=\mathcal{P}\left(\alpha_{1}, \ldots, \alpha_{k}\right)$, where $\langle\mathcal{R}\rangle$ consists of all finite sums $P_{1} * Q_{1}+\ldots+P_{m} * Q_{m}\left(m \in \mathbb{N} ; Q_{1}, \ldots Q_{m} \in \mathcal{R} ; P_{1}, \ldots, P_{m} \in M_{k}^{+}\right)$. Define $\mathcal{A}(\mathcal{R})$ as follows:

$$
\mathcal{A}(\mathcal{R})=\operatorname{span}\left\{F_{i}=\bigoplus_{Q \in \mathcal{R}} Q^{1 / 2} E_{i i} Q^{-1 / 2}: 1 \leq i \leq k\right\}
$$

Since $\mathcal{P}\left(\alpha_{1}, \ldots, \alpha_{k}\right)$ is the unit ball of some norm on $\mathbb{C}^{k}$ we have that there exists $\delta>0$ so that $\left|w_{i}\right|<\delta$ implies $\left(w_{1}, \ldots, w_{k}\right) \in \mathcal{P}\left(\alpha_{1}, \ldots, \alpha_{k}\right)$. Thus, for all $\left|w_{i}\right|<\delta$ and for all $\left(q_{i j}\right) \in \mathcal{R}$ we have that $\left(\left(1-\bar{w}_{i} w_{j}\right) q_{i j}\right) \geq 0$. This yields $\left(q_{i j}\right) \geq\left(\bar{w}_{i} q_{i j} w_{j}\right)$ for all $\left|w_{i}\right|<\delta$ and for all $\left(q_{i j}\right) \in \mathcal{R}$. Integrating this inequality around the torus of radius $\delta$ yields $\left(q_{i j}\right) \geq \delta^{2} \operatorname{Diag}\left(q_{i i}\right)$ for all $\left(q_{i j}\right) \in \mathcal{R}$. Thus, $\delta^{2} q_{m m} E_{m m} \leq\left(q_{i j}\right)$ for each $1 \leq m \leq k$ and for each $\left(q_{i j}\right) \in \mathcal{R}$. Hence if $F_{i}=\bigoplus_{Q \in \mathcal{R}} Q^{1 / 2} E_{i i} Q^{-1 / 2}$, then $F_{i}^{*} F_{i}=\bigoplus_{Q \in \mathcal{R}} Q^{-1 / 2} q_{i i} E_{i i} Q^{-1 / 2} \leq \delta^{-2} \bigoplus_{Q \in \mathcal{R}} I_{k \times k}$ and we have that $\left\|F_{i}\right\| \leq \delta^{-1}$. Thus each $F_{i}$ is bounded and $\mathcal{A}(\mathcal{R})$ is a $k$-idempotent operator algebra.

Lemma A. $\mathcal{S}_{n}(\mathcal{A}(P))=\mathcal{S}_{n}(\mathcal{A}(\mathcal{R}))$ for all $n \geq 1$. 
Proof. We have the following logical equivalences:

$$
\begin{aligned}
\left\|\sum_{i=1}^{k} w_{i} F_{i}\right\| \leq 1 & \Longleftrightarrow\left\|\bigoplus_{Q \in \mathcal{R}} Q^{1 / 2} D_{w} Q^{-1 / 2}\right\| \leq 1 \\
& \Longleftrightarrow\left(\left(1-\bar{w}_{i} w_{j}\right) q_{i j}\right) \geq 0 \text { for all } Q \in \mathcal{R} \\
& \Longleftrightarrow\left(w_{1}, \ldots, w_{k}\right) \in \mathcal{P}\left(\alpha_{1}, \ldots, \alpha_{k}\right) \\
& \Longleftrightarrow\left\|\sum_{i=1}^{k} w_{i} E_{i}\right\| \leq 1 .
\end{aligned}
$$

Therefore $\mathcal{A}(\mathcal{R})$ is isometrically isomorphic to $\mathcal{A}(P)$. Hence $\mathcal{A}(\mathcal{R})$ is completely isometrically isomorphic to $\mathcal{A}(P)$ (see Section 3). Thus by Theorem $4, \mathcal{S}_{n}(\mathcal{A}(P))=$ $\mathcal{S}_{n}(\mathcal{A}(\mathcal{R}))$ for all $n \geq 1$.

Lemma B. $\mathcal{S}_{n}\left(\mathcal{A}(P)^{*} \mathcal{A}(P)\right) \subseteq \mathcal{S}_{n}\left(\mathcal{A}(\mathcal{R})^{*} \mathcal{A}(\mathcal{R})\right)$ for all $n \geq 1$.

Proof. By Theorem $3, \mathcal{S}_{n}\left(\mathcal{A}(P)^{*} \mathcal{A}(P)\right) \subseteq \mathcal{S}_{n}\left(\mathcal{A}(\mathcal{R})^{*} \mathcal{A}(\mathcal{R})\right)$ for all $n \geq 1$ if and only if the map $F_{i}^{*} F_{j} \longrightarrow E_{i}^{*} E_{j}$ is completely positive. The map $\pi: \mathcal{A}(P) \longrightarrow$ $\bigoplus_{Q \in \mathcal{R}} M_{k}$, where $\pi\left(E_{i}\right)=F_{i}$, is a complete isometry (Lemma A) and by a theorem of M. Hamana [6] there exists a $*$-epimorphism $\gamma_{2}$ such that the following diagram commutes:

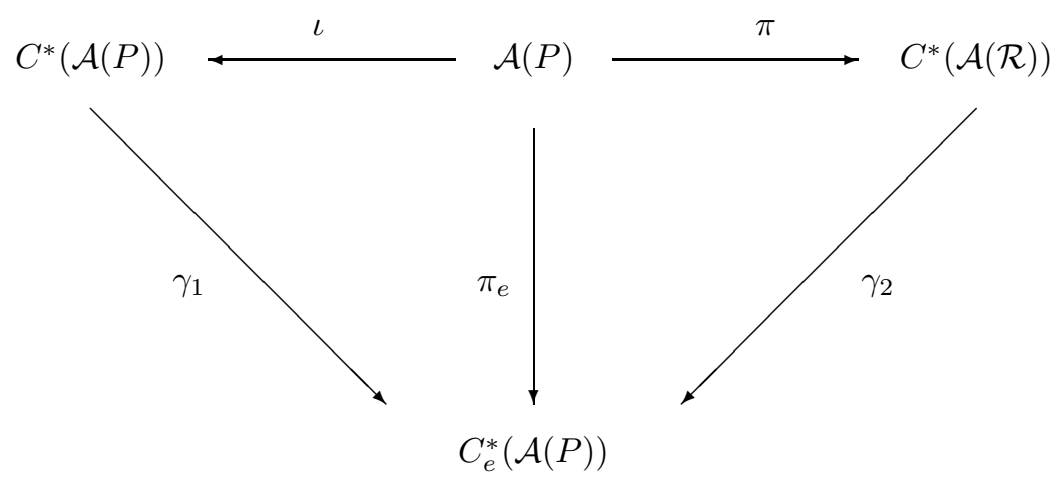

In the above diagram $C^{*}(\mathcal{A}(P))$ denotes the $C^{*}$-algebra generated by the idempotents $E_{1}, \ldots, E_{k}$ and $C^{*}(\mathcal{A}(\mathcal{R}))$ is defined analogously. Also, $C_{e}^{*}(\mathcal{A}(P))$ denotes the $C^{*}$-envelope of the operator algebra $\mathcal{A}(P)$. The $C^{*}$-envelope of an operator algebra $\mathcal{B}$ is a $C^{*}$-quotient of any $C^{*}$-algebra that contains $\mathcal{B}$ completely isometrically. Thus the $C^{*}$-envelope is in a precise sense the "minimal" $C^{*}$-algebra generated by $\mathcal{B}$. The concept of the $C^{*}$-envelope is due to W. Arveson [2] and M. Hamana [6].

Note that $C^{*}(\mathcal{A}(P))$ is $*$-isomorphic to $M_{k}$, but $M_{k}$ is simple and hence $C^{*}(\mathcal{A}(P))$ must be $C_{e}^{*}(\mathcal{A}(P))$. Thus we have that $\gamma_{1}$ is a $*$-isomorphism. If we define $\gamma: C^{*}(\mathcal{A}(\mathcal{R})) \longrightarrow C^{*}(\mathcal{A}(P))$ to be $\gamma=\gamma_{1}^{-1} \circ \gamma_{2}$, then $\gamma$ is a $*$-epimorphism. Therefore $\gamma$ is completely positive and $\mathcal{S}_{n}\left(\mathcal{A}(P)^{*} \mathcal{A}(P)\right) \subseteq \mathcal{S}_{n}\left(\mathcal{A}(\mathcal{R})^{*} \mathcal{A}(\mathcal{R})\right)$ for all $n \geq 1$ by Theorem 3 .

Lemma C. $\mathcal{S}_{1}\left(\mathcal{A}(\mathcal{R})^{*} \mathcal{A}(\mathcal{R})\right)=\langle\mathcal{R}\rangle^{-}$. 
Proof. We will first show that $\langle\mathcal{R}\rangle^{-} \subseteq \mathcal{S}_{1}\left(\mathcal{A}(\mathcal{R})^{*} \mathcal{A}(\mathcal{R})\right)$. Let $\left(r_{i j}\right) \in\langle\mathcal{R}\rangle^{-}$. We must show that the map $\phi: \mathcal{A}(\mathcal{R})^{*} \mathcal{A}(\mathcal{R}) \longrightarrow \mathbb{C}$, defined by $\phi\left(F_{i}^{*} F_{j}\right)=r_{i j}$, is completely positive. Note that:

$$
\begin{aligned}
\left(a_{i j} F_{i}^{*} F_{j}\right) \geq 0 & \Longleftrightarrow\left(a_{i j} q_{i j} E_{i j}\right) \geq 0 \text { for all } Q \in \mathcal{R} \\
& \Longleftrightarrow\left(a_{i j} q_{i j}\right) \geq 0 \text { for all } Q \in \mathcal{R} .
\end{aligned}
$$

Let $\sum_{i, j} a_{i j} F_{i}^{*} F_{j} \geq 0$. By Lemma $1, \sum_{i, j} a_{i j} F_{i}^{*} F_{j} \geq 0$ if and only if $\left(a_{i j} F_{i}^{*} F_{j}\right) \geq 0$. Consequently $\sum_{i, j} a_{i j} F_{i}^{*} F_{j} \geq 0$ if and only if $\left(a_{i j} q_{i j}\right) \geq 0$ for all $Q \in \mathcal{R}$. Thus $\left(a_{i j} r_{i j}\right) \geq 0$ which implies that $\sum_{i, j} a_{i j} r_{i j}=\phi\left(\sum_{i, j} a_{i j} F_{i}^{*} F_{j}\right) \geq 0$. Hence $\phi$ is a positive linear functional. Since $\mathcal{A}(\mathcal{R})^{*} \mathcal{A}(\mathcal{R})$ is an operator system, $\phi$ is completely positive and $\left(q_{i j}\right) \in \mathcal{S}_{1}\left(\mathcal{A}(\mathcal{R})^{*} \mathcal{A}(\mathcal{R})\right)$.

We shall now show that $\mathcal{S}_{1}\left(\mathcal{A}(\mathcal{R})^{*} \mathcal{A}(\mathcal{R})\right) \subseteq\langle\mathcal{R}\rangle^{-}$. Suppose that

$$
\left(\widehat{q}_{i j}\right) \in \mathcal{S}_{1}\left(\mathcal{A}(\mathcal{R})^{*} \mathcal{A}(\mathcal{R})\right)
$$

and $\left(\widehat{q}_{i j}\right) \notin\langle\mathcal{R}\rangle^{-}$. Since $\langle\mathcal{R}\rangle^{-}$is a cone in $M_{k}$ we can pick a bounded linear functional $f: M_{k} \longrightarrow \mathbb{C}$ such that $f\left(\langle\mathcal{R}\rangle^{-}\right) \geq 0$ and $f\left(\left(\widehat{q}_{i j}\right)\right)<0$, where $f\left(E_{i j}\right)=$ $a_{i j}$. Thus,

$$
\begin{aligned}
f(R)=\sum_{i, j=1}^{k} a_{i j} r_{i j} \geq 0 \text { for all } R \in \mathcal{R} \Longleftrightarrow & \left(a_{i j} r_{i j}\right) \geq 0 \text { for all } \\
& R \in \mathcal{R} \text { by Prop. } 5 \\
\Longleftrightarrow & \left(a_{i j} \otimes F_{i}^{*} F_{j}\right) \geq 0 \\
\Longleftrightarrow & \left(a_{i j} \otimes q_{i j}\right) \geq 0 \text { for all } \\
& \left(q_{i j}\right) \in \mathcal{S}_{1}\left(\mathcal{A}(\mathcal{R})^{*} \mathcal{A}(\mathcal{R})\right) \\
\Longrightarrow & \sum_{i, j=1}^{k} a_{i j} q_{i j} \geq 0 \text { for all } \\
& \left(q_{i j}\right) \in \mathcal{S}_{1}\left(\mathcal{A}(\mathcal{R})^{*} \mathcal{A}(\mathcal{R})\right) .
\end{aligned}
$$

But this contradicts the fact that $f\left(\left(\widehat{q}_{i j}\right)\right)<0$ since $f\left(\left(\widehat{q}_{i j}\right)\right)=\sum_{i, j=1}^{k} a_{i j} \widehat{q}_{i j} \geq 0$. Therefore $\mathcal{S}_{1}\left(\mathcal{A}(\mathcal{R})^{*} \mathcal{A}(\mathcal{R})\right) \subseteq\langle\mathcal{R}\rangle^{-}$and $\mathcal{S}_{1}\left(\mathcal{A}(\mathcal{R})^{*} \mathcal{A}(\mathcal{R})\right)=\langle\mathcal{R}\rangle^{-}$.

Proof of Theorem A. Recall that $\mathcal{S}_{n}(\mathcal{A}(P))=\mathcal{S}_{n}\left(\mathcal{A}(P)^{*} \mathcal{A}(P)\right)$ for all $n \geq 1$ and that $\mathcal{S}_{1}\left(\mathcal{A}(P)^{*} \mathcal{A}(P)\right)=\langle P\rangle$ (Section 3). We also have that $\mathcal{S}_{n}\left(\mathcal{A}(\mathcal{R})^{*} \mathcal{A}(\mathcal{R})\right) \subseteq$ $\mathcal{S}_{n}(\mathcal{A}(\mathcal{R}))$ for all $n \geq 1$ via Theorem 4 .

Lemma B states that $\mathcal{S}_{n}\left(\mathcal{A}(P)^{*} \mathcal{A}(P)\right) \subseteq \mathcal{S}_{n}\left(\mathcal{A}(\mathcal{R})^{*} \mathcal{A}(\mathcal{R})\right)$ for all $n \geq 1$ and Lemma A states that $\mathcal{S}_{n}(\mathcal{A}(P))=\mathcal{S}_{n}(\mathcal{A}(\mathcal{R}))$ for all $n \geq 1$. But this implies that $\mathcal{S}_{n}\left(\mathcal{A}(P)^{*} \mathcal{A}(P)\right)=\mathcal{S}_{n}\left(\mathcal{A}(\mathcal{R})^{*} \mathcal{A}(\mathcal{R})\right)$ for all $n \geq 1$. Thus by Lemma $\mathrm{C}$ we have that

$$
\langle P\rangle=\mathcal{S}_{1}\left(\mathcal{A}(P)^{*} \mathcal{A}(P)\right)=\mathcal{S}_{1}\left(\mathcal{A}(\mathcal{R})^{*} \mathcal{A}(\mathcal{R})\right)=\langle\mathcal{R}\rangle^{-} .
$$

Therefore, $\mathcal{I}=\left\langle\left(\frac{1}{1-\bar{\alpha}_{i} \alpha_{j}}\right)\right\rangle$.

\section{ACKNOWLEDGEMENTS}

This work is taken from the author's Ph.D. thesis, done under the supervision of Professor Vern I. Paulsen. The author wishes to express his gratitude to Professor Paulsen for his guidance and introducing the idea of studying the "duality" of hyperconvex sets which led to this work. 


\section{REFERENCES}

[1] J. Agler, Interpolation, preprint.

[2] W.B. Arveson, Subalgebras of $C^{*}$-Algebras II, Acta Math. 123 (1972), 271-308. MR 52:15035

[3] F. Bonsall and J. Duncan, Complete Normed Algebras, Springer-Verlag, New York/Berlin, 1973. MR 54:11013

[4] B. Cole, K. Lewis and J. Wermer, Pick Conditions on a Uniform Algebra and von Neumann Inequalities, J. Functional Anal. 107 (1992), 235-254. MR 93e:46059]

[5] B. Cole and J. Wermer, Pick Interpolation, von Neumann Inequalities, and Hyperconvex sets, Complex Potential Theory, P.M. Gauthier (ed.), NATO Adv. Sci. Inst. Ser. C 439, Kluwer, Dordrecht, (1994), 89-129. MR 96m:46092

[6] M. Hamana, Injective envelopes of Operator Systems, Publ. RIMS, Kyoto Univ. 15 (1979), 773-785. MR 81h:46071

[7] R. Nevanlinna, Ueber beschrankte Funktionen, die in gegebene Punkten vorgeschriebene Werte annehmen, Ann. Acad. Sci. Fenn. (1919), 1-71.

[8] R. Nevanlinna, Ueber beschrankte analytische Funktionen, Ann. Acad. Sci. Fenn. \#7 32 (1929).

[9] V.I. Paulsen, Matrix-valued interpolation and hyperconvex sets, Int. Eqn. and Op. Thy., to appear.

[10] V.I. Paulsen, Operator algebras of idempotents, preprint.

[11] G. Pick, Ueber die Beschraenkungen analytisher Funktionen, welche durch vorgegebene Funktionswete bewirkt werden, Math. Ann. 77 (1916), 7-23.

[12] D. Sarason, Generalized interpolation in $H^{\infty}$, Trans. Amer. Math. Soc. 127 (1967), 179-203. MR 34:8193

[13] E.L. Stout, The Theory of Uniform Algebras, Bogden and Quigley, New York, 1971. MR 54:11066

[14] A.T. Tomerlin, Products of Nevanlinna-Pick Kernels and Operator Colligations, Int. Eq. and Op. Thy. 38, 2000, 350-356. CMP 2001:05

Department of Mathematics, University of Georgia, Athens, Georgia 30602

E-mail address: solazzo@math.uga.edu 